Supplement of Biogeosciences Discuss., 11, 16799-16824, 2014

http://www.biogeosciences-discuss.net/11/16799/2014/

doi:10.5194/bgd-11-16799-2014-supplement

(C) Author(s) 2014. CC Attribution 3.0 License.

(c) (1)

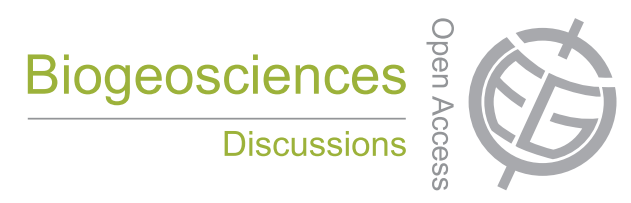

Supplement of

\title{
Distribution of black carbon in Ponderosa pine litter and soils following the High Park wildfire
}

\author{
C. M. Boot et al.
}

Correspondence to: C. M. Boot (claudia.boot@ colostate.edu) 
1 Table S1. Results of three-way ANOVA evaluating the effects of layer, burn intensity and 2 slope on $\mathrm{C}$ and $\mathrm{N}$ values.

\begin{tabular}{|c|c|c|c|c|c|c|c|c|c|c|c|}
\hline \multirow[b]{2}{*}{ response variable } & \multirow[b]{2}{*}{ df } & \multicolumn{2}{|c|}{$\log \% C$} & \multicolumn{2}{|c|}{$\log \% \mathrm{~N}$} & \multicolumn{2}{|c|}{$\mathrm{C}: \mathrm{N}$} & \multicolumn{2}{|c|}{$\log C$ stock } & \multicolumn{2}{|c|}{ bulk density } \\
\hline & & f-value & $p$-value & $\mathrm{f}$-value & $p$-value & f-value & $p$-value & f-value & $p$-value & f-value & $p$-value \\
\hline slope & 2 & 1.09 & 0.342 & 0.11 & 0.897 & 3.99 & 0.022 & 0.16 & 0.853 & 0.05 & 0.955 \\
\hline burn & 2 & 7.82 & $<0.001$ & 1.46 & 0.239 & 25.87 & $<0.001$ & 2.38 & 0.099 & 0.13 & 0.874 \\
\hline layer & 2 & 273.45 & $<0.001$ & 182.27 & $<0.001$ & 98.14 & $<0.001$ & 21.93 & $<0.001$ & 2.22 & 0.142 \\
\hline slope x burn & 4 & 0.21 & 0.934 & 0.32 & 0.861 & 0.15 & 0.961 & 0.46 & 0.762 & 0.48 & 0.751 \\
\hline slope $\mathrm{x}$ layer & 4 & 0.85 & 0.496 & 0.78 & 0.544 & 0.37 & 0.833 & 0.40 & 0.807 & 0.41 & 0.664 \\
\hline burn x layer & 4 & 5.24 & $<0.001$ & 1.30 & 0.278 & 21.52 & $<0.001$ & 0.89 & 0.476 & 0.19 & 0.827 \\
\hline slope $\mathrm{x}$ burn $\mathrm{x}$ layer & 8 & 0.00 & 1 & 0.47 & 0.874 & 0.50 & 0.853 & 0.61 & 0.770 & 0.26 & 0.905 \\
\hline
\end{tabular}

3

4

Table S2. Results of post-hoc comparisons evaluating the source of significant differences in $\% \mathrm{C}, \% \mathrm{~N}, \mathrm{C}: \mathrm{N}$ and $\mathrm{C}$ stocks by burn, layer, slope and the interaction terms, not significant (ns), not tested (nt).

\begin{tabular}{|c|c|c|c|c|c|c|c|c|}
\hline \multirow[b]{2}{*}{ post hoc comparisons } & \multicolumn{2}{|c|}{$\log \% C$} & \multicolumn{2}{|c|}{$\log \% \mathrm{~N}$} & \multicolumn{2}{|c|}{$\log C: N$} & \multicolumn{2}{|c|}{$\log C$ stock } \\
\hline & diff. means & $p$ value & diff. means & $p$ value & diff. means & $p$ value & diff. means & $p$ value \\
\hline high vs. unburned & 0.19 & $<0.001$ & "ns & ns & 7.22 & $<0.001$ & ns & ns \\
\hline moderate vs. unburned & 0.07 & 0.146 & ns & ns & 4.02 & $<0.001$ & ns & ns \\
\hline high vs. moderate & 0.13 & 0.017 & ns & ns & 3.20 & 0.002 & ns & ns \\
\hline $0-5 \mathrm{~cm}$ vs litter & 0.79 & $<0.001$ & 0.58 & $<0.001$ & 11.73 & $<0.001$ & 0.38 & $<0.001$ \\
\hline 5-15 cm vs litter & 1.10 & $<0.001$ & 0.87 & $<0.001$ & 12.40 & $<0.001$ & 0.41 & $<0.001$ \\
\hline $0-5 \mathrm{~cm}$ vs $5-15 \mathrm{~cm}$ & 0.31 & $<0.001$ & 0.29 & $<0.001$ & 0.67 & 0.501 & 0.03 & 0.696 \\
\hline $0-5$ vs $5-15$ degrees & nt & nt & nt & nt & 2.29 & 0.051 & nt & nt \\
\hline $0-5$ vs $15-30$ degrees & nt & nt & nt & nt & 2.63 & 0.031 & nt & nt \\
\hline $5-15 \mathrm{~cm}$ vs $15-30$ degress & nt & nt & nt & nt & 0.35 & 0.722 & nt & nt \\
\hline litter: unburned vs. moderate & 0.196 & 0.024 & 0.196 & 0.024 & 13.48 & $<0.001$ & 0.17 & 0.277 \\
\hline litter: unburned vs. high & 0.492 & $<0.001$ & 0.492 & $<0.001$ & 19.96 & $<0.001$ & 0.14 & 0.21 \\
\hline litter: moderate vs. high & 0.296 & 0.001 & 0.296 & 0.001 & 6.48 & $<0.001$ & 0.31 & 0.02 \\
\hline $0-5 \mathrm{~cm}$ : unburned vs. moderate & 0.057 & 0.755 & ns & ns & 1.55 & 0.609 & 0.02 & 0.831 \\
\hline $0-5 \mathrm{~cm}$ : unburned vs. high & 0.0562 & 0.511 & ns & ns & 0.221 & 0.899 & 0.08 & 0.759 \\
\hline $0-5 \mathrm{~cm}$ : moderate vs. high & 0.113 & 0.439 & ns & ns & 1.774 & 0.654 & 0.10 & 0.753 \\
\hline 5-15 cm: unburned vs. moderate & 0.0561 & 0.883 & ns & ns & 0.139 & 0.937 & 0.07 & 0.803 \\
\hline 5-15 cm: unburned vs. high & 0.021 & 0.805 & ns & ns & 1.483 & 0.78 & 0.09 & 0.808 \\
\hline 5-15 cm: moderate vs. high & 0.0351 & 0.893 & ns & ns & 1.344 & 0.675 & 0.02 & 0.831 \\
\hline
\end{tabular}


13 Table S3. Results of three-way ANOVA evaluating the effects of layer, burn intensity and

14 slope on BPCA-C concentration and stocks.

\begin{tabular}{|c|c|c|c|c|c|c|}
\hline \multirow[b]{2}{*}{ response variable } & \multicolumn{3}{|c|}{$\begin{array}{c}\text { BPCA C ( } \mathrm{g}^{*} \mathrm{~kg}^{-1} \text { organic } \\
\text { carbon) }\end{array}$} & \multicolumn{3}{|c|}{$\begin{array}{c}\text { BPCA C stock (g BPCA-C } \\
* \mathrm{~m}^{-2} \text { ) }\end{array}$} \\
\hline & df & f-value & $p$-value & df & f-value & $p$-value \\
\hline slope & 2 & 1.04 & 0.357 & 2 & 0.41 & 0.663 \\
\hline burn & 2 & 5.37 & 0.007 & 2 & 4.70 & 0.012 \\
\hline layer & 2 & 0.62 & 0.541 & 2 & 18.07 & $<0.001$ \\
\hline slope x burn & 4 & 1.46 & 0.223 & 4 & 0.27 & 0.894 \\
\hline slope $\mathrm{x}$ layer & 4 & 0.52 & 0.724 & 4 & 0.27 & 0.894 \\
\hline burn x layer & 4 & 7.85 & $<0.001$ & 4 & 7.10 & $<0.001$ \\
\hline slope $\mathrm{x}$ burn $\mathrm{x}$ layer & 8 & 0.76 & 0.637 & 8 & 0.49 & 0.861 \\
\hline
\end{tabular}

16 Table S4. Results of post-hoc comparisons evaluating the source of significant differences

17 in BPCA-C concentration and stocks by burn, layer, and the burn by layer interaction.

\begin{tabular}{|c|c|c|c|c|}
\hline \multirow[b]{2}{*}{ post hoc comparisons } & \multicolumn{2}{|c|}{$\begin{array}{l}\text { BPCA C (g BPCA-C * } \\
\mathrm{kg}^{-1} \text { organic carbon) }\end{array}$} & \multicolumn{2}{|c|}{$\begin{array}{l}\text { BPCA C stock (g } \\
\text { BPCA-C } * m^{-2} \text { ) }\end{array}$} \\
\hline & diff. means & $p$ value & diff. means & $\mathrm{p}$ value \\
\hline high vs. unburned & 5.66 & 0.005 & 0.21 & 0.096 \\
\hline moderate vs. unburned & 3.76 & 0.071 & 0.32 & 0.010 \\
\hline high vs. moderate & 1.90 & 0.271 & 0.11 & 0.288 \\
\hline $0-5 \mathrm{~cm}$ vs litter & 1.83 & 0.655 & 0.58 & $<0.001$ \\
\hline $5-15 \mathrm{~cm}$ vs litter & 1.50 & 0.632 & 0.50 & $<0.001$ \\
\hline $0-5 \mathrm{~cm}$ vs $5-15 \mathrm{~cm}$ & 0.33 & 0.850 & 0.08 & 0.388 \\
\hline unburned: $0-5 \mathrm{~cm}$ vs litter & 7.85 & $<0.001$ & 1.25 & $<0.001$ \\
\hline unburned: $5-15 \mathrm{~cm}$ vs litter & 7.68 & 0.003 & 1.17 & $<0.001$ \\
\hline unburned: $0-5 \mathrm{~cm}$ vs $5-15 \mathrm{~cm}$ & 0.18 & 0.440 & 0.08 & 0.667 \\
\hline moderate: $0-5 \mathrm{~cm}$ vs litter & 6.93 & 0.073 & 0.19 & 0.653 \\
\hline moderate: $5-15 \mathrm{~cm}$ vs litter & 3.68 & 0.385 & 0.17 & 0.593 \\
\hline moderate: $0-5 \mathrm{~cm}$ vs $5-15 \mathrm{~cm}$ & 3.25 & 0.287 & 0.02 & 0.916 \\
\hline high: $0-5 \mathrm{~cm}$ vs litter & 7.85 & 0.028 & 0.30 & 0.263 \\
\hline high: $5-15 \mathrm{~cm}$ vs litter & 7.68 & 0.022 & 0.29 & 0.194 \\
\hline high: $0-5 \mathrm{~cm}$ vs $5-15 \mathrm{~cm}$ & 0.18 & 0.953 & $<0.01$ & 0.978 \\
\hline litter: unburned vs. moderate & 14.84 & $<0.001$ & 1.06 & $<0.001$ \\
\hline litter: unburned vs. high & 18.38 & $<0.001$ & 0.82 & $<0.001$ \\
\hline litter: moderate vs. high & 3.54 & 0.234 & 0.24 & 0.181 \\
\hline $0-5 \mathrm{~cm}$ : unburned vs. moderate & 1.36 & 0.881 & 0.14 & 0.828 \\
\hline $0-5 \mathrm{~cm}:$ unburned vs. high & 1.99 & 0.885 & 0.13 & 0.706 \\
\hline 0-5 cm: moderate vs. high & 0.63 & 0.832 & $<0.01$ & 0.964 \\
\hline 5-15 cm: unburned vs. moderate & 2.20 & 0.731 & 0.09 & 0.943 \\
\hline 5-15 cm: unburned vs. high & 2.80 & 0.736 & 0.05 & 0.945 \\
\hline 5-15 cm: moderate vs. high & 0.60 & 0.844 & 0.04 & 0.828 \\
\hline
\end{tabular}


19 Table S5. Results of two way ANOVA evaluating the effects of burn and layer on the

20 relative abundances of B6CA, B5CA, B4CA and the ratio of B4CA:B6CA and B5CA:B6CA.

\begin{tabular}{|l|r|r|r|r|r|r|r|r|r|}
\hline & & \multicolumn{2}{c|}{ B6CA } & \multicolumn{2}{c|}{ B5CA } & \multicolumn{2}{c|}{ B4CA } & \multicolumn{2}{c|}{ B5CA:B6CA } \\
\hline response variable & df & f-value & $p$-value & f-value & $p$-value & f-value & $p$-value & -value & $p$-value \\
\hline \hline burn & 2 & 0.30 & 0.918 & 0.05 & 0.95 & 0.82 & 0.444 & 0.13 & 0.876 \\
\hline layer & 2 & $\mathbf{1 . 9 4}$ & $<\mathbf{0 . 0 0 1}$ & $\mathbf{2 7 . 5 7}$ & $<\mathbf{0 . 0 0 1}$ & $\mathbf{1 3 . 9 6}$ & $<\mathbf{0 . 0 0 1}$ & $\mathbf{1 7 . 5 6 3}$ & $<\mathbf{0 . 0 0 1}$ \\
\hline burn x layer & 4 & 2.53 & 0.059 & 1.02 & 0.4 & 0.84 & 0.504 & 1.242 & 0.299 \\
\hline
\end{tabular}

21

22 Table S6. Post hoc comparisons among layers (litter, 0-5 $\mathrm{cm}$ soils, and 5-15 $\mathrm{cm}$ soils) for

23 relative abundance of $\mathrm{B} 6 \mathrm{CA}, \mathrm{B} 5 \mathrm{CA}, \mathrm{B} 4 \mathrm{CA}$ and the ratio of $\mathrm{B} 5 \mathrm{CA}: \mathrm{B} 6 \mathrm{CA}$.

\begin{tabular}{|l|r|r|r|r|r|r|r|r|r|}
\hline & \multicolumn{2}{|c|}{ B6CA } & \multicolumn{2}{c|}{ B5CA } & \multicolumn{2}{c|}{ B4CA } & \multicolumn{2}{c|}{ B5CA:B6CA } \\
\hline post hoc comparisons & diff. means & $p$ value & diff. means & $p$ value & diff. means & $p$ value & diff. means & $p$ value \\
\hline \hline $0-5 \mathrm{~cm}$ vs litter & $<0.001$ & 1.000 & $\mathbf{7 . 1 7}$ & $<\mathbf{0 . 0 0 1}$ & $\mathbf{8 . 2 1}$ & $<\mathbf{0 . 0 0 1}$ & 0.142 & 0.066 \\
\hline $5-15 \mathrm{~cm}$ vs litter & $\mathbf{8 . 9 8}$ & $<\mathbf{0 . 0 0 1}$ & $\mathbf{1 0 . 4 8}$ & $<\mathbf{0 . 0 0 1}$ & 2.88 & 0.073 & $\mathbf{0 . 3 7}$ & $<\mathbf{0 . 0 0 1}$ \\
\hline $0-5 \mathrm{~cm}$ vs 5-15 cm & $\mathbf{8 . 9 8}$ & $<\mathbf{0 . 0 0 1}$ & $\mathbf{3 . 3 1}$ & $\mathbf{0 . 0 2 4}$ & $\mathbf{5 . 3 3}$ & $\mathbf{0 . 0 0 2}$ & $\mathbf{0 . 2 2 8}$ & $\mathbf{0 . 0 0 1}$ \\
\hline
\end{tabular}

24

25 